\title{
Optimization Design of Inner Structure and Material of Cars Front Bumper Based on Finite Element Method
}

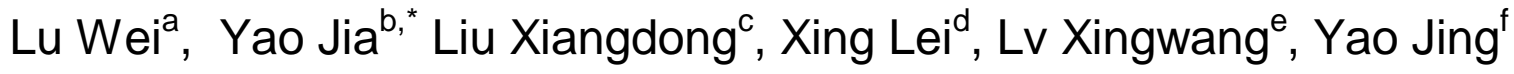 \\ College of Mechanical Engineering, Jiamusi University, 154007, Jiamusi, China \\ alujungi2001@163.com, biahuiyuyao@163.com, cliuxiangdong1973@126.com,

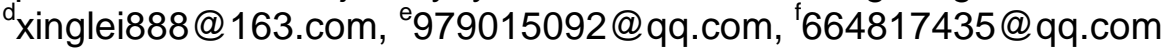 \\ *Corresponding author: jiahuiyuyao@163.com
}

\begin{abstract}
Keywords: Bumper Design; Collision Analysis; Optimized Design; Lightweight.
Abstract. The ideal design of the bumper should take full advantage of the bumper plastic deformation to absorb most crash energy in automobile collisions. In the paper, the bumpers with different inner structure (circular cross section form and honeycomb cross section), different materials (Al alloy, structural steel and polyethylene) have been researched by finite element method. The simulation of the bumper deformation in the collision process through simulation calculation has been obtained. The collision resistance of the bumper has been evaluated, and the cross section shape and material parameters have been optimized. When the simulation method used for the enterprise design bumper, the design cycle, test times and costs saving can be reduced effectively.
\end{abstract}

\section{Introduction}

In recent years, the number of casualties in traffic accidents increased, making the increasing automobile manufacturers and consumers emphasis on vehicle safety issues. As the limits of real car crash test subjecting for longer time and higher cost consuming, generally only a small number of confirmatory tests have been carried. In the actual product design, finite element analysis methods have been extensive use to replace the crash tests and research results indicating the use of ANSYS software to simulate the real vehicle collision test has good agreement results with the real test, which can effectively substitute vehicle crash tests to conduct collision analysis. The ideal design of the bumper should take full advantage of the bumper plastic deformation to absorb most crash energy in automobile collisions [1]. Car bumpers are usually simplified to thin-walled structures, therefore, the optimized design of the bumper structure can improve the collision energy absorbing characteristics [2].

For the purpose of the car bumper can absorb more energy and also realize the principle of lightweight design in the collision process, we adopt hollow bumper support structure in the bumper sandwich wall to reduce its own weight, realize cost savings, and enhance energy absorption effect. This paper studies the impact of different shape cross-sections (circle shape and cellular structure) and material properties effect on the energy absorption characteristics of the car bumper, using Pro-E design software to build a three-dimensional finite element analysis of bumper collision model. And the collision simulation and optimization design has been carried out, thus the bumper with the optimal collision energy absorption characteristics has been obtained.

\section{Research method}

Based on a comprehensive review and analysis of the key problems, research methods and general situations of passive safety technologies of car crash at home and abroad, according to the bumper collision standard, also considering integrity, comprehensive and intuitive characteristics of the actual working situation, the vehicle model is established, through the simulation of collision situation, the comprehensive analysis of the front bumper touch resistance of the vehicle at low speed collision has been conduct, the detailed flow chart of the main work done in this paper is as follows in Fig.1: 


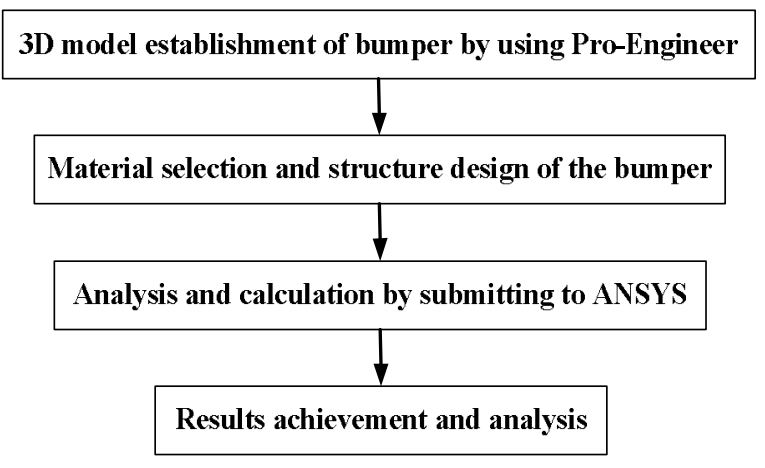

Fig. 1 The working process chart

\section{D modeling and simulation analysis}

3D modeling of circular and honeycomb shape cross section form. In Pro-E software, the establishment of 3D entity model with $1700 \mathrm{~mm}$ long, $210 \mathrm{~mm}$ high, $50 \mathrm{~mm}$ thickness of the U-shaped bumper has been set up. Establishing a hollow cylindrical entity and a six prism entity of $50 \mathrm{~mm}$ outer diameter, $40 \mathrm{~mm}$ inner diameter and $40 \mathrm{~mm}$ height, and the hollow entities are assembled to the inner of the bumper shell, finally array processing of the entities has been assembled into a bumper shell have been done, to get the needed model. The two types of hollow inner structures are as shown in Fig. 2 (a) and (b).

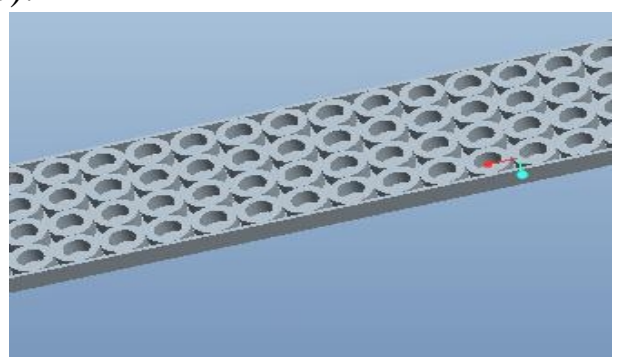

(a) circular cross section form

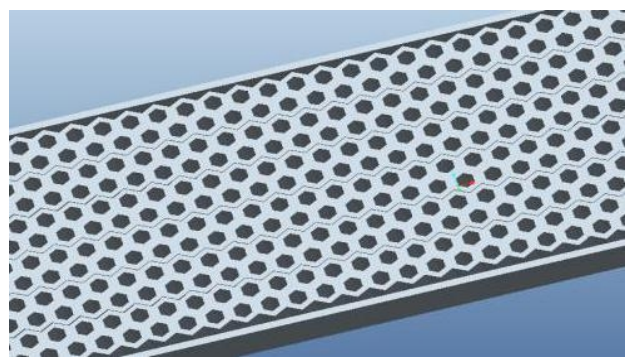

(b) honeycomb shape cross section form

Fig. 2 Hollow inner structures of bumper

The finite element analysis of the bumper. By using import interface, introducing 3D entity model into ANSYS, and meshing the model, the meshing result is shown in Fig. 3, then click the model to carry on the interface analysis, uniform positive pressure $10000 \mathrm{~N}$ is forced. The fixed constraints imposed on the left and right sides of ear parts to ensure $\mathrm{X}, \mathrm{Z}$ axis fixed, and $\mathrm{Y}$ direction is in free state as in Fig. 4.

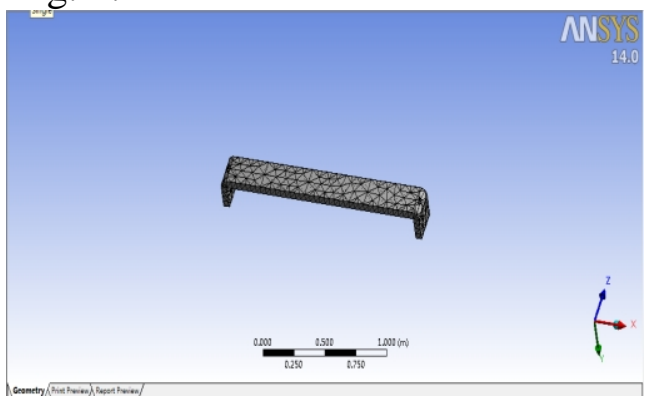

Fig. 3 Model meshing

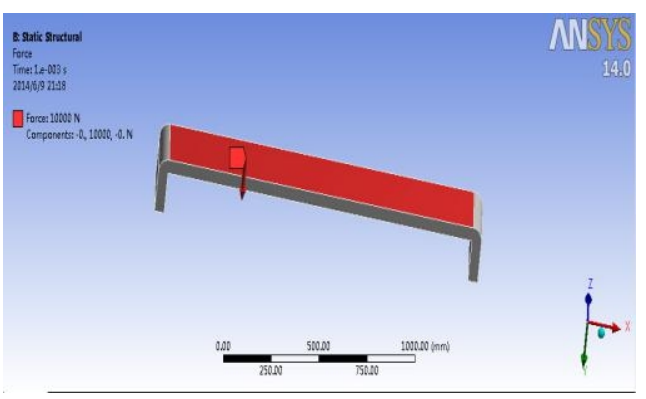

Fig. 4 Applied load and constraints

Comparison of circular and honeycomb cross section of $\mathrm{Al}$ alloy material bumper. Under the finite element software condition, to simulate the stress and deformation of the two kinds of bumper, the result is shown in Fig. 5 and 6. Von Mises (the equivalent stress) is a kind of yield criterion; it follows the material mechanics (theory) of the fourth strength theory. When maximum shear stress (particle) reaches a certain value, the object will yield. The mathematical expressions [3] are shown as Eq.1: 


$$
\left|\sigma_{\max }-\sigma_{\min }\right|=\sigma_{s}=2 K
$$

Where $\mathrm{K}$ is the shear yield strength, $\boldsymbol{\sigma}_{\max }, \boldsymbol{\sigma}_{\min }$ are the maximum value and minimum value of equivalent stress.

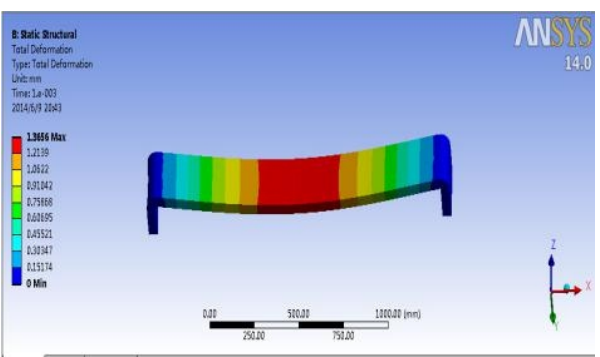

(a) Total deformation

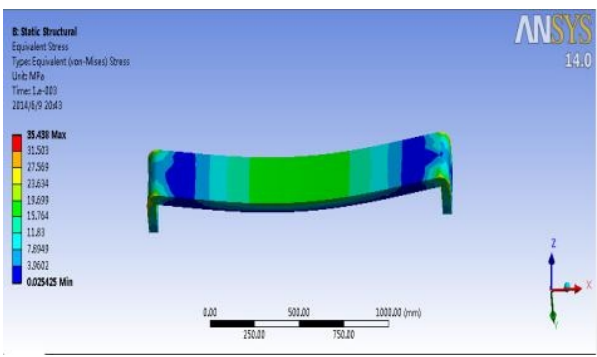

(b) Equivalent stress

Fig. 5 Total deformation and equivalent stress of circular cross section form

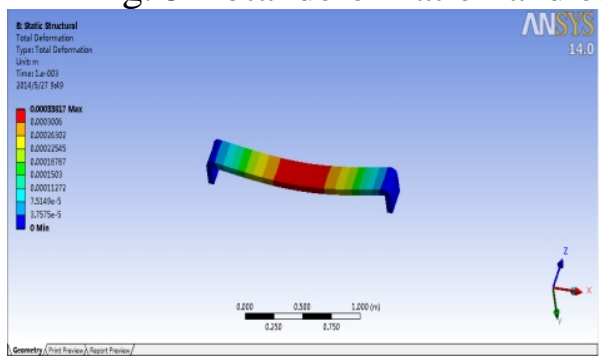

(a) Total deformation

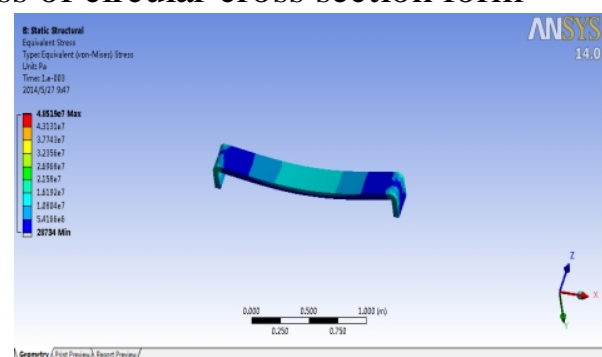

(b) Equivalent stress

Fig. 6 Total deformation and equivalent stress of honeycomb shape cross section form

Table 1 The results comparison table

\begin{tabular}{ccc}
\hline & Circular cross section & Honeycomb cross section \\
\hline Total deformation $(\mathrm{mm})$ & 1.3656 & 0.34769 \\
Shear yield strength $(\mathrm{MPa})$ & 35.413 & 35.3947 \\
\hline
\end{tabular}

Shear yield strength data in Table 1 were got according to Eq. 1 and the maximum and minimum equivalent stress values in Fig.5 and Fig.6. Through the comparisons of the two kinds of model simulation in Table 1, the total deformation of honeycomb shape cross section is smaller, but the yield strength of the circular cross section and honeycomb cross section bumper are relatively equal, so the energy absorption effect of honeycomb shape cross section is better.

\section{Modal analysis of Al alloy material of two different cross section shape of bumper}

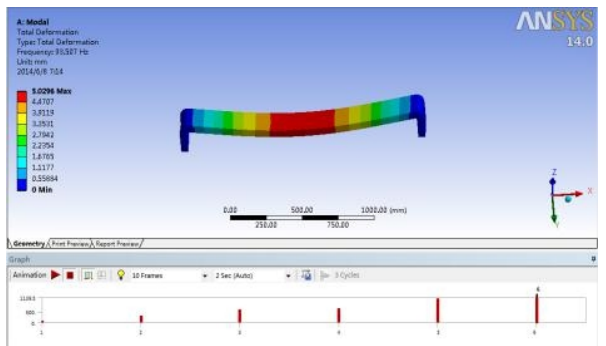

(a) Circular cross section

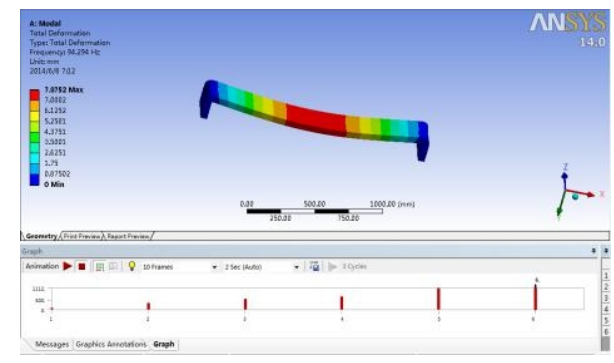

(b) Honeycomb cross section

Fig. 7 Modal analysis of circular cross section form and honeycomb shape cross section form

The so-called modal analysis can determine natural frequency and vibration mode of the design structure and mechanical parts, which can be taken as the important parameters, can provide the references for the optimization design for the vibration characteristic analysis of the structural system, the vibration fault diagnosis and the structural dynamic characteristics. The frequency of the object is related to its hardness, the size, quality, when the deformation happens, elastic characteristic makes it recovery. Elasticity is mainly related to size and hardness, the mass affects the acceleration. When the 
shapes are the same, high hardness has high frequency, high mass has low frequency. By using the Modal analysis of ANSYS module, the two different cross section shape of bumper have been analyzed, analysis effect is as in Fig.7.

Table 2 The results comparison of six-order modal

\begin{tabular}{ccccccc}
\hline & 1 & 2 & 3 & 4 & 5 & 6 \\
\hline Circular cross section (Hz) & 93.507 & 306.54 & 595.41 & 640.43 & 1075.7 & 1139.5 \\
Honeycomb cross section (Hz) & 94.294 & 316.24 & 532.02 & 663.25 & 1076.7 & 1112 \\
\hline
\end{tabular}

From 6 order natural vibration frequency values in Table 2, the honeycomb shape cross section of the bumper can be seen is relatively larger. When the shapes are the same, high hardness has high frequency, high mass has low frequency. So a honeycomb structure is relatively higher hardness, and the strength of the honeycomb cross section bumper are relatively high and the safety coefficient is larger.

Table 3 The results comparison of different materials

\begin{tabular}{cccc}
\hline & Al alloy & Structural steel & Polyethylene \\
\hline Total deformation $(\mathrm{mm})$ & 0.34769 & 0.52154 & 61.543 \\
Shear yield strength $(\mathrm{MPa})$ & 35.3947 & 39.5827 & 26.0816 \\
\hline
\end{tabular}

Through the simulation comparison of the bumper with $\mathrm{Al}$ alloy, structural steel and polyethylene, total deformation and shear yield strength of the honeycomb shaped cross section are in Table $3, \mathrm{Al}$ alloy minimizes the total deformation of bumpers, but the total deformation of polyethylene bumper is largest, the shear yield strength of structural steel bumper is the largest, the shear yield strength of polyethylene bumper is largest. So the result of comprehensive consideration of lightweight and mechanical characteristics, the energy absorption effect of $\mathrm{Al}$ alloy material bumper with honeycomb cross section is better.

\section{Conclusions}

In this paper, on the basis of summarizing the predecessors' work, the bumpers with different inner structure (circular cross section form and honeycomb cross section), different materials (Al alloy, structural steel and polyethylene) have been researched by the finite element analysis, the simulation of the bumper deformation in the collision process is obtained. The collision resistance of the bumper has been evaluated, and the cross section shape and material parameters on the crash worthiness of the bumper system have been analyzed. The energy absorption effect of honeycomb cross section made by $\mathrm{Al}$ alloy bumper is the best, which can not only improve the bumper energy absorbing characteristic, but also reduce the deformation of the bumper. When the simulation method is used for the enterprise design bumper, the design cycle can be shorten, test times and costs saving can also be reduced effectively.

\section{Acknowledgments}

This project was supported by the Youth Backbone Support Plan for Heilongjiang Province Universities (1254G059) and the Innovation team of Jiamusi University (Cxtd-2016-02).

\section{References}

[1] B.G. Li, X.K. Chen, Y. Lin, Crashworthiness and Structural Optimization of Front Bumper, J. Jilin University. 39(1) (2009) 12-16.

[2] J.C. Li, X.S. Cheng, Capacity enhancement of impact energy absorbing member of vehicle, Automotive Engineering, 30(11) (2008) 984-986. 
[3] Y.S. Yang, Car bumper system low-speed collision performance study, Harbin: Harbin Engineering University, Master degree theses. (2009). 